\title{
Determinantes do hábito de fumar e de seu abandono durante a gestação em localidade urbana na região sul do Brasil ${ }^{*}$
}

\section{Determining factors related to smoking and its abandonment during pregnancy in an urban locality in Southern Brazil}

\author{
Iná S. Halal**, Cesar G. Victora**, Fernando C. Barros**
}

\begin{abstract}
HALAL, I. S. et al. Determinantes do hábito de fumar e de seu abandono durante a gestação em localidade urbana na região sul do Brasil. Rev. Saúde Pública, 27: 105-12, 1993. Foi realizado um estudo transversal, com 873 gestantes que freqüentaram o pré-natal em Pelotas (RS), em 1989-90, com o objetivo de investigar possiveis fatores de risco e. fatores prognósticos para o tabagismo durante a gravidez. A prevalência no início da gravidez foi de $40,8 \%$. O hábito de fumar da mãe da gestante e do marido e a baixa escolaridade da mulher estiveram associados com o risco de fumar no início da gravidez. O tabagismo do marido esteve associado com um aumento de cerca de duas vezes nesse risco. A taxa de abandono até a $15^{\mathrm{a}}-22^{3}$ semana gestacional foi de $35,6 \%$. A renda familiar, o hábito de fumar da mãe da gestante e do companheiro, a idade de início, duração e intensidade do hábito da mulher estiveram associados com a interrupção durante a gravidez. Os resultados acima permaneceram após ajuste para fatores de confusão, através de análise estratificada.
\end{abstract}

Descritores: Fumo, epidemiologia. Gravidez. Fatores socioeconômicos.

\section{Introdução}

Os dados disponíveis na literatura tornam inequívocos os malefícios do hábito de fumar da mãe durante a gestação sobre a saúde fetal, aumentando o risco de ter um recém-nascido com baixo peso e/ou pequeno para a idade gestacional $1^{5,13}$, bem como de parto prematuro ${ }^{2,3,5}$ e de mortalidade perinatal ${ }^{12,14}$.

Embora o percentual de fumantes tenha diminuico em alguns países do mundo a partir dos anos 60 , o número absoluto de mulheres que fumam atualmente é muito maior do que em décadas passadas ${ }^{11}$. No entanto, estudos têm demonstrado não apenas ser freqüente a interrupção espontânea do hábito no início da gestação como também as melhorias das condições fetais decorrentes desta interrupção ${ }^{1,10,13,16}$. Os grupos maternos de maior risco, entretanto, precisam ser melhor conhecidos para que medidas preventivas efctivas possam ser

* Pesquisa subvencionada pelo "Intemational Development Research Center", do Canadá, apresentado em Sessão de Comunicação Coordenada no II Congresso Brasileiro de Epidemiologia, julho de 1992, Belo Horizonte, MG - Brasil.

** Departamento de Medicina Social da Universidade Federal de Pelotas - Pelotas, RS. Brasil

Separatas/Reprints: I. S. Halal - Av. Duque de Caxias, 250 Fragata - Caixa Postal 464 - 96030-000 Pelotas, RS. Brasil. instituídas. Dessa forma, o presente estudo visa a detectar fatores de risco e fatores prognósticos associados com o hábito de fumar no início e durante a gravidez.

\section{Material e Método}

As informações aqui apresentadas fazem parte do "Estudo de Prevenção de Resultados Perinatais Desfavoráveis Através do Apoio Psicossocial Durante a Gravidez". Este estudo randomizado controlado multicêntrico, foi realizado no período de março de 1989 a fevereiro de 1991 nas cidades de Rosário (Argentina), México (México), Havana (Cuba) e Pelotas (Brasil), esta última analisada no presente trabalho.

Pelotas é uma cidade com cerca de 300.000 habitantes, em que $90 \%$ da assistência pré-natal concentra-se em três clínicas pré-natais do Instituto Nacional do Seguro Social ${ }^{15}$. A população alvo do estudo foi composta de gestantes residentes na zona urbana de Pelotas, com idade gestacional entre 15 e 22 semanas e que compareceram ao prénatal nesses três serviços, no período de julho de 1989 a julho de 1990. Havendo dúvida quanto à idade gestacional, aceitava-se como critério substitutivo a altura uterina entre 15 e $20 \mathrm{~cm}$, inclusive, com base no registro da carteira da gestante.

As seguintes variáveis foram coletadas por meio de entrevistas com a população estudada: 
idade, paridade, raça, altura, peso, história reprodutiva, data da última menstruação, estado marital, educação, hábito de fumar no início da gestação e no periodo da entrevista, renda familiar mensal e estrutura familiar.

A pesquisa foi estruturada com um delineamento transversal; e, uma vez que as mulheres foram identificadas com 15 a 22 semanas de gestação e inquiridas sobre o hábito de fumar no início da gravidez, o estudo possui também um elemento de coorte retrospectiva. As hipóteses eram de que a idade inferior a 25 anos, o nível socioeconômico baixo (escolaridade, renda, aglomeração doméstica), ausência do companheiro e o hábito de fumar dos pais da gestante e do companheiro (quando presente) seriam fatores de risco quanto ao hábito de fumar no inicio da gestação. Estes mesmos fatores de risco, juntamente com a longa duração, a maior intensidade e a idade precoce de início do hábito seriam fatores prognósticos que dificultariam seu abandono durante a gestação. Pressupondo-se uma prevalência de exposição aos fatores de risco de $30 \%$, estimou-se que uma amostra de cerca de mil gestantes, um terço das quais fumantes, seria suficiente para detectar um risco relativo de 1,5 ou mais significativo ao nível de 5\% (bi-caudal), com $80 \%$ de poder estatístico.

Por outro lado, supondo que $30 \%$ das 300 gestantes fumantes abandonariam o hábito durante a gestação, o estudo teria um poder de $85 \%$ de detectar um risco relativo de 2,0 para uma exposição aos supostos fatores prognósticos.

$\mathrm{O}$ trabalho de campo foi realizado por seis assistentes sociais que entrevistavam as gestantes no serviço de pré-natal em que estas consultavam, com autorização prévia do chefe do serviço.

As assistentes sociais foram treinadas antes de iniciarem o trabalho de campo e participaram da fase piloto do estudo. Durante esta fase, redigiu-se um detalhado manual de instruçōes sobre a coleta de dados para ser usado ao longo do estudo.

O teste qui-quadrado foi usado para avaliar a significância estatística das associaçð̄es entre fatores de risco e hábito de fumar no início da gestação e entre os fatores prognósticos e abandono do hábito durante a gestação?

A magnitude das associações foi avaliada através do cálculo das razōes de prevalências (riscos relativos) e de seus intervalos de confiança. Quando havia possibilidade de confusão, utilizou-se análise estratificada de Mantel-Haenszel para investigar mais detalhadamente o efeito do fator de risco e do fator prognóstico sobre o desfecho?

\footnotetext{
* Intervalo de Confiança
}

\section{Resultados}

A amostra foi constituída por 873 gestantes que preencheram os critérios descritos acima. Não houve recusas. Quando não foi possível obter-se informação para alguma variável isolada, a entrevistada foi excluida somente das análises correspondentes.

As características sociodemográficas das gestantes estão a seguir descritas. A média de idade foi de 25,6 anos, com maior concentração (31\%) no grupo etário de 20 a 24 anos. Cerca de $20 \%$ das gestantes eram adolescentes e $11 \%$ tinham 35 anos ou mais. A renda familiar de $37,7 \%$ foi menor ou igual a dois salários mínimos. As gestantes estudadas tinham em média 5,9 anos completos de escola, sendo que $84 \%$ tinham menos de 9 anos e um terço menos de 5 anos. Quanto à aglomeração, $28 \%$ das gestantes relataram que em suas casas dormiam 3 ou mais pessoas por quarto. Cerca de $90 \%$ viviam com marido ou companheiro, das quais $58 \%$ relataram que os mesmos eram fumantes. A história de tabagismo (atual ou no passado) do pai da gestante foi positiva para $72 \%$ das mulheres, e da mãe para $32 \%$.

A prevalência de tabagismo no início da gravidez foi de $41 \%$. Por ocasião da entrevista, $36 \%$ destas haviam abandonado o hábito, sendo a prevalência nesse período de $27 \%$.

Ressalte-se que quatro mulheres passaram a fumar depois de iniciada a gestação. Estas foram incluídas na análise como pertencendo ao grupo das atuais fumantes.

As principais características do hábito de fumar entre as mulheres que fumavam no início da gravidez estão descritas a seguir: $29 \%$ iniciaram a fumar antes dos 14 anos de idade, sendo a idade média de início igual a 15,3 anos (desvio padrão = 3,7 ; mediana $=15$ ). O número médio de cigarros fumados por dia no início da gravidez foi de 12,7 $(s=8,8 ; m=10)$, sendo que $44 \%$ consumiam mais de 10 cigarros por dia. Em relação à duração, $40 \%$ das fumantes tinham o hábito há mais de 10 anos; e 73\% há mais de 5 . A duração média do hábito de fumar foi de 10,1 anos $(s=6,4 ; m=9)$.

Cento e vinte e oito mulheres pararam de fumar entre o início da gestação e a $15^{\mathrm{a}}-22^{\mathrm{a}}$ semana, equivalendo a uma taxa de abandono de $36 \%$. Entre as 232 que continuaram fumando, o número médio de cigarros consumidos por dia decresceu de $13,4(s=8,5 ; m=10)$ no início da gestação a $8,5(\mathrm{~s}=7,1 ; \mathrm{m}=6)$ entre a $15^{\mathrm{a}}-22^{\mathrm{a}}$ semana. A redução média foi de $-4,9$ cigarros por dia $\left(s=7,8 ; I C^{*} 95 \%\right.$ de $\left.3,74-6,04\right)$. O teste $t$ emparelhado foi significativo ao nivel de $p<0,001$ bicaudal $(t=-9,28)$. No início da gravidez, $45 \%$ das fumantes consumiam mais do que 
10 cigarros por dia; na $15^{\mathrm{a}}-22^{\mathrm{a}}$ semana, este percentual reduziu-se para apenas $21 \%$.

A Tabela 1 mostra a prevalência de tabagismo entre as gestantes no início da gravidez, de acordo com as variáveis independentes. Em termos de idade, a prevalência foi ligeiramente maior entre as adolescentes (44\%), seguidas pelas mulheres de 25 a 29 anos (43\%); estas diferenças não foram significativas $(p=0,7)$. As razōes de prevalências nos diferentes grupos etários, tomando as mulheres de 20 a 24 anos como grupo de referência (Tabela 1), mostram que em todos os grupos etários o intervalo de confiança de $95 \%$ incluiu a unidade.

O tabagismo no início da gravidez mostrou clara associação inversa com a escolaridade (Tabela 1): as gestantes sem escolaridade formal apresentaram prevalência quase $80 \%$ superior àquelas com 9 ou mais anos de estudo $(\mathrm{p}<0,01)$. Por outro lado, não houve associação entre tabagismo e renda, cohabitação e aglomeração doméstica (Tabela 1).

A Tabela 2 mostra ser o tabagismo quase duas vezes mais comum entre as gestantes cujo marido ou companheiro fumava do que entre as demais $(p=0,001 ;$ IC $95 \%$ de 1,52 a 2,26$)$.
A história de tabagismo do pai da gestante apresentou uma associação limitrofe $(p=0,06$; $\mathrm{RP}=1,21$ IC $95 \%=0,99-1,47)$. As filhas de mães fumantes, por outro lado, apresentaram risco $28 \%$ maior de serem fumantes quando comparadas com as demais ( $p=0,004)$.

$O$ ajuste do efeito dessas variáveis para escolaridade, pelo método de Mantel-Haenszel, não implicou razões de prevalências substancialmente diferentes dos valores brutos (Tabela 2 ).

As Tabelas 3, 4 e 5 mostram a análise da taxa de abandono do hábito de fumar do início da gestação até a $15^{\mathrm{a}}-22^{\mathrm{a}}$ semana. A maior freqüuência de abandono ocorreu entre as mulheres com 35 anos ou mais $(47 \%)$, seguidas imediatamente pelas de 30 a 34 anos.

Para o grupo de gestantes mais idosas, o limite inferior do IC foi igual à unidade, embora a associação global não fosse significativa $(p=0,3)$. A renda familiar (Tabela 3 ) apresentou associação direta com $o$ abandono do hábito $(\mathbf{p}<0,001)$ : gestantes com renda familiar mensal superior a dois salários mínimos tiveram uma probabilidade $64 \%$ maior de abandonar quando comparadas com as mais pobres. Não houve as-

Tabela 1. Prevalência do hábito de fumar no inicio da gravidez e razão de prevalências conforme variáveis independentes $(N=873$ ). Pelotas, $R S, 1989-90$.

\begin{tabular}{|c|c|c|c|c|}
\hline Caracteristica & $N$ & $\begin{array}{l}\text { Prevalência do } \\
\text { Hábito de } \\
\text { Fumar }\end{array}$ & $\begin{array}{l}\text { Razâo de } \\
\text { Prevalências }\end{array}$ & $I C(95 \%)$ \\
\hline
\end{tabular}

idade em anos

$\begin{array}{rrrrr}<20 & 163 & 44,2 \% & 1,11 & 0,89-1,39 \\ 20-24 & 269 & 39,8 \% & 1,00 & \\ 25-29 & 192 & 42,7 \% & 1,07 & 0,86-1,34 \\ 30-34 & 153 & 39,9 \% & 1,00 & 0,79-1,28 \\ >=35 & 96 & 35,4 \% & 0,89 & 0,65-1,21\end{array}$

Anos de escola completos

$\begin{array}{rr}0 & 28 \\ 1-4 & 259 \\ 5-8 & 443 \\ >=9 & 143\end{array}$

$53,6 \%$

$46,3 \%$

$40,2 \%$

$30,1 \%$
1,78

1,54

1,34

1,00
$1,16-2,73$

$1,16-2,04$

$1,02 \cdot 1,76$

Renda familiar

$$
\begin{array}{ll}
>2 S M & 541 \\
<=2 S M & 329
\end{array}
$$

$38,6 \%$

$44,4 \%$

1,00

1,15

$0,98-1,35$

Vive com marido ou companheiro

Sim $\quad 774$

Número de pessoas por quarto

$$
1-2 \quad 625
$$

IC - Intervalo de Confiança 
Tabela 2. Prevalência do hábito de fumar no inicio da gravidez e razão de prevalências conforme variáveis independentes, controladas para escolaridade materna ( $N=873)$. Pelotas, RS, $1989-90$.

\begin{tabular}{|c|c|c|c|c|}
\hline \multirow{2}{*}{ Característica } & \multirow{2}{*}{$\mathbf{N}$} & \multirow{2}{*}{$\begin{array}{l}\text { Prevalência do } \\
\text { Hábito de Fumar }\end{array}$} & \multicolumn{2}{|c|}{ Razão de Prevalências } \\
\hline & & & Bruta (IC* 95\%) & Ajustada (IC 95\%) \\
\hline \multicolumn{5}{|c|}{ Companheiro Fumante } \\
\hline $\operatorname{Sim}_{\text {Não }}$ & $\begin{array}{l}445 \\
329\end{array}$ & $\begin{array}{l}50,1 \% \\
27,1 \%\end{array}$ & $\begin{array}{l}1,85(1,52-2,26) \\
1,00\end{array}$ & $\begin{array}{l}1,83(1,50-2,23) \\
1,00\end{array}$ \\
\hline
\end{tabular}

Pai Fumante

$\begin{array}{lllll}\text { Sim } & 613 & 42,7 \% & 1,21(0,99-1,47) & 1,14(0,94-1,40) \\ \text { Não } & 237 & 35,4 \% & 1,00 & 1,00\end{array}$

Mãe Fumante

\begin{tabular}{cllll}
$\operatorname{Sim}$ & 276 & $47,8 \%$ & $1,28(1,09-1,51)$ & $1,25(1,07-1,47)$ \\
Não & 593 & $37,3 \%$ & 1,00 & 1,00 \\
\hline
\end{tabular}

IC - Intervalo de Confiança

Tabela 3. Taxa do abandono do hábito de fumar até a $15^{\mathrm{a}}-22^{\mathrm{a}}$ semana de gravidez conforme algumas variáveis sociodemográficas quantitativas $(N=360)$. Pelotas, $R S, 1989$ - 90 .

\begin{tabular}{|c|c|c|c|c|}
\hline Característica & $N$ & $\begin{array}{c}\text { Taxa de abandono } \\
\text { do Hábito de } \\
\text { Fumar }\end{array}$ & $\begin{array}{l}\text { Razâo de } \\
\text { Prevalências }\end{array}$ & $1 C(95 \%)$ \\
\hline \multicolumn{5}{|l|}{ idade em anos } \\
\hline $\begin{array}{r}<20 \\
20-24 \\
25-29 \\
30-34 \\
>=35\end{array}$ & $\begin{array}{c}74 \\
108 \\
83 \\
61 \\
34\end{array}$ & $\begin{array}{l}36,5 \% \\
33,3 \% \\
28,9 \% \\
41,0 \% \\
47,1 \%\end{array}$ & $\begin{array}{l}1,26 \\
1,15 \\
1,00 \\
1,42 \\
1,63\end{array}$ & $\begin{array}{l}0,80-1,98 \\
0,75-1,77 \\
0,90-2,23 \\
1,00-2,66\end{array}$ \\
\hline
\end{tabular}

Anos de escola completos

$\begin{array}{ccccc}0-4 & 138 & 35,5 \% & 1,00 & \\ 5-8 & 179 & 35,2 \% & 0,99 & 0,73-1,34 \\ >=9 & 43 & 37,2 \% & 1,05 & 0,67-1,64\end{array}$

Renda familiar

$\begin{array}{ccccc}>2 \text { SM } & 209 & 42,6 \% & 1,64 & 1,20-2,24 \\ <=2 \text { SM } & 150 & 26,0 \% & 1,00 & \end{array}$

Vive com marido ou companheiro

\begin{tabular}{|c|c|c|c|c|}
\hline $\begin{array}{l}\text { Sim } \\
\text { Não }\end{array}$ & $\begin{array}{c}316 \\
44\end{array}$ & $\begin{array}{l}34,8 \% \\
40,9 \%\end{array}$ & $\begin{array}{l}0,85 \\
1,00\end{array}$ & $0,58-1,25$ \\
\hline \multicolumn{5}{|c|}{ ssoas por quarto } \\
\hline $\begin{array}{l}1-2 \\
>=3\end{array}$ & $\begin{array}{l}248 \\
112\end{array}$ & $\begin{array}{l}34,8 \% \\
40,9 \%\end{array}$ & $\begin{array}{l}1,35 \\
1,00\end{array}$ & $0,97-1,89$ \\
\hline
\end{tabular}

IC - Intervalo de Confiança

sociação significativa entre abandono do hábito e escolaridade, cohabitação e aglomeração doméstica.

A Tabela 4 indica que 0 abandono foi cerca de 1,5 vezes mais freqüente entre as gestantes cujo companheiro não fumava $(p=0,03)$. As filhas de mães não-fumantes apresentaram uma chance $60 \%$ maior de abandonarem quando comparadas com filhas de mães fumantes, mas o mesmo não foi observado para o tabagismo do pai. 
Tabela 4. Taxa de abandono do hábito de fumar até a $15^{\mathrm{a}}-22^{\mathrm{a}}$ semana de gravidez conforme variáveis sociodemográficas qualitativas, ajustadas para renda familiar $(N=360)$, Pelotas, $R S, 1989-90$

\begin{tabular}{|c|c|c|c|c|}
\hline \multirow{2}{*}{ Característica } & \multirow{2}{*}{$\mathrm{N}$} & \multirow{2}{*}{$\begin{array}{c}\text { Taxa de Abando- } \\
\text { no do Hábito de } \\
\text { Fumar }\end{array}$} & \multicolumn{2}{|c|}{ Razão de Prevalência } \\
\hline & & & Bruta (IC 95\%) & Ajustada (IC 95\%) \\
\hline \multicolumn{5}{|c|}{ Companheiro Fumante } \\
\hline $\begin{array}{l}\text { Sim } \\
\text { Não }\end{array}$ & $\begin{array}{c}226 \\
90\end{array}$ & $\begin{array}{l}31,0 \% \\
44,4 \%\end{array}$ & $\begin{array}{l}1,00 \\
1,43(1,06-1,94)\end{array}$ & $\begin{array}{l}1,00 \\
1,44(1,07-1,94)\end{array}$ \\
\hline \multicolumn{5}{|l|}{ Pai Fumante } \\
\hline $\begin{array}{l}\text { Sim } \\
\text { Não }\end{array}$ & $\begin{array}{c}265 \\
85\end{array}$ & $\begin{array}{l}34,3 \% \\
40,0 \%\end{array}$ & $\begin{array}{l}1,00 \\
1,16(0,86-1,59)\end{array}$ & $\begin{array}{l}1,00 \\
1,11(0,82-1,51)\end{array}$ \\
\hline \multicolumn{5}{|l|}{ Māe Fumante } \\
\hline $\begin{array}{l}\text { Sim } \\
\text { Não }\end{array}$ & $\begin{array}{l}134 \\
223\end{array}$ & $\begin{array}{l}26,1 \% \\
41,7 \%\end{array}$ & $\begin{array}{l}1,00 \\
1,60(1,15-2,21)\end{array}$ & $\begin{array}{l}1,00 \\
1,56(1,13-2,15)\end{array}$ \\
\hline
\end{tabular}

Tabela 5. Taxa de abandono do hábito de fumar até a $15^{\mathrm{a}}-22^{\mathrm{a}}$ semana de gravidez conforme algumas características do hábito da mulher, ajustadas para renda familiar ( $N=356)$. Pelotas, $R S, 1989-90$.

\begin{tabular}{lcccc}
\hline \multirow{2}{*}{ Caracteristica } & $\mathrm{N}$ & $\begin{array}{c}\text { Taxa de Abandono } \\
\text { do Hábito de }\end{array}$ & \multicolumn{2}{c}{ Razão de Prevaléncias } \\
\cline { 5 - 6 } & & Fumar & Bruta (IC 95\%) & Ajustada (IC 95\%) \\
\hline Idade em anos com que & & & & \\
iníciou a fumar & & & & \\
$7-13$ & 103 & $25,2 \%$ & 1,00 & 1,00 \\
14 ou mais & 257 & $39,7 \%$ & $1,57(1,09-2,26)$ & $1,47(1,02-2,12)$ \\
Duração do hábito em anos & & & & \\
$0-5$ & & & & \\
$6-10$ & 99 & $42,4 \%$ & $0,97(0,68-1,37)$ & $0,97(0,69-1,36)$ \\
$11-15$ & 117 & $31,6 \%$ & $0,72(0,50-1,05)$ & $0,74(0,51-1,07)$ \\
16 ou mais & 71 & $23,9 \%$ & $0,55(0,33-0,89)$ & $0,56(0,35-0,91)$ \\
Número de cigarros por dia & 73 & $43,8 \%$ & 1,00 & 1,00 \\
no inicio da gravidez & & & & \\
$1-5$ & & & & \\
$6-10$ & 95 & $52,6 \%$ & $1,58(1,18-2,12)$ & $1,63(1,22-2,16)$ \\
11 ou mais & 105 & $24,8 \%$ & $0,74(0,50-1,11)$ & $0,77(0,51-1,14)$ \\
& 156 & $33,3 \%$ & 1,00 & 1,00 \\
\hline
\end{tabular}

A Tabela 5 mostra as taxas de interrupção conforme as características do hábito de fumar no início da gestação. A taxa de abandono entre as mulheres que fumavam no máximo cinco cigarros por dia no início da gravidez foi de $53 \%$, contra $33 \%$ nas que fumavam mais de dez $(p<0,001)$. As fumantes cujo hábito iniciou com 14 anos ou mais de idade apresentaram uma tendência para o abandono $60 \%$ superior a das demais fumantes ( $p$ $=0,013$ ). Houve também maiores taxas de interrupção entre as que fumavam há cinco anos ou menos e entre aquelas que fumavam há dezesseis anos ou mais $(p=0.02)$ (Tabela 5). Examinandose simultaneamente os efeitos da idade de início e da duração, ficou evidente que, para todas as categorias de duração, o abandono foi maior entre as que haviam iniciado a fumar após os 14 anos. Observou-se, ainda, uma discreta redução na taxa de abandono com maiores durações do hábito, com exceção das mulheres que fumavam há mais de 15 anos. Este subgrupo apresentava uma média de idade superior aos demais subgrupos, o que pode 
explicar sua maior taxa de abandono, uma vez que, conforme mostrado na Tabela 3 , as taxas de abandono aumentaram com a idade. $O$ ajuste para renda do efeito das demais variáveis não modificou substancialmente as razões de prevalências de abandono (Tabelas 4 e 5).

\section{Discussăo}

O presente estudo envolveu 873 gestantes que frequientaram os três principais serviços públicos de tratamento pré-natal da cidade de Pelotas, no período de um ano. Não foram obtidas, portanto, informaçס̋es de gestantes que utilizaram serviços privados. O pré-natal público, em Pelotas, responde por cerca de $90 \%$ da assistência pré-natal, sendo, além disso, alta a percentagem de mulheres que consultam durante a gravidez ( $95 \%$ ), embora cerca de $15 \%$ o façam somente após o quinto mês ${ }^{8}$. Assim, embora a amostra não seja representativa de todas as gestantes de Pelotas daquele ano, mesmo assim cobriu cerca de $70 \%$ de toda a demanda do período. Por outro lado, o interesse principal do estudo não era de extrapolar os resultados para toda a cidade, mas sim fazer comparações internas.

A restrição do estudo a determinadas idades gestacionais (de 15 a 22 semanas) poderia ser afetada por falta de precisão. Para evitar este viés, a altura uterina foi utilizada como critério substitutivo quando a mãe não lembrava a data da última menstruação.

Cerca de $4: \%$ das gestantes eram fumantes quando se iniciou a gravidez. Esta prevalência é consistente com os achados de Victora e col. ${ }^{15}$ que encontraram para a cidade de Pelotas, no ano de $1982,36 \%$ de fumantes entre as parturientas. Outros países apresentaram resultados semelhantes: no estudo de Kuzma e Kissinger ${ }^{9}$ na California (EUA), a prevalência de tabagismo entre mais de 12.000 gestantes foi de $35 \%$; e, Rubin e col. ${ }^{12}$ na Dinamarca, relataram uma freqüência de $40 \%$.

A escolaridade apresentou a associação linear inversa com o hábito de fumar no início da gestação; quanto menor o número de séries escolares cursadas maior a proporção de fumantes. No estudo de Kuzma e Kissinger ${ }^{9}$ eram fumantes $59,9 \%$ das gestantes com menos de 9 anos de escolaridade, contra $12,8 \%$ daquelas que tinham 17 ou mais anos de instrução formal. Em Pelotas, em 1982, 54\% das analfabetas eram fumantes contra $30 \%$ das mulheres com 9 anos ou mais de escolaridade. O presente achado, portanto, é compatível com a literatura.

Outras variáveis sociodemográficas como idade, renda, ausência do companheiro e aglomeração doméstica não apresentaram associação com o hábito de fumar no início da gravidez.
Houve associação estatisticamente significativa com o hábito de fumar do marido e da mãe e com a escolaridade da gestante. $O$ hábito de fumar do pai apresentou uma associação limiar. As variáveis hábito de fumar do marido e da mãe da gestante mantiveram-se significativamente associadas com o hábito de fumar no início da gravidez, mesmo após controle para a escolaridade da mulher. A maior probabilidade dos filhos de pais fumantes tornarem-se também fumantes, observada por outros autores ${ }^{4,6}$, foi detectada em nosso estudo principalmente em relação ao tabagismo da mãe. Assim, filhas de mães fumantes apresentaram risco $28 \%$ maior de fumarem quando comparadas com as controles. Um achado bastante interessante diz respeito à associação encontrada entre o tabagismo da gestante e de seu companheiro. Eram fumantes $50 \%$ das gestantes cujos companheiros fumavam, contra $27 \%$ das demais, ou seja, mulheres que viviam com fumantes apresentaram um risco de fumar $85 \%$ maior, quando comparadas com as controles. Confirmando este achado, o estudo de Rubin e col. ${ }^{12}$ demonstrou que $46 \%$ dos homens fumaram durante a gravidez de suas companheiras, tendo havido naquele estudo uma correlação altamente significativa entre hábito de fumar da mulher e hábito de fumar do companheiro (coeficiente de correlação $=0,25 ; \mathrm{p}<0,0001$ ).

No período compreendido entre a $15^{\mathrm{a}}$ e a $22^{\mathrm{a}}$ semana de gravidez, cerca de $36 \%$ das $356 \mathrm{mu}$ lheres que fumavam no início haviam interrompido o hábito. Com isto, a prevalência do tabagismo na $15^{\mathbf{2}}-22^{\mathbf{3}}$ semana de gravidez foi de $27 \%$. Dados ainda não publicados do estudo multicêntrico mostraram uma prevalência semelhante em Cuba (27\%) e na Argentina (22\%), contra uma frequiência excepcionalmente baixa $(5.5 \%)$ no México, para iguais períodos gestacionais. Outros resultados deste mesmo estudo mostraram que, mais tarde, na $36^{4}$ semana da gestação as prevalências de tabagismo não eram substancialmente diferentes destas: 25\% em Cuba, 19\% na Argentina e $4 \%$ no México. No Brasil, os dados disponiveis para mulheres incluídas no estudo multicêntrico (56\% da amostra de nossa investigação), mostraram para o grupo controle uma prevalência de $33 \%$ na $15^{\mathrm{z}}-22^{\mathrm{z}}$ semana contra $35 \%$ na $36^{\mathrm{a}}$. Estes resultados são consistentes com o achado de Sexton e Hebel ${ }^{13}$ de que mulheres que pararam de fumar espontaneamente antes do segundo trimestre da gravidez, tendem a não voltar a fumar até o parto; e de que os abandonos espontâneos depois do primeiro trimestre são raros. Isto sugere que o presente estudo, embora restrito ao primeiro trimestre, incluiu efetivamente 0 período em que ocorreram mudanças no hábito de fumar das gestantes. 
As variáveis que apresentaram associação estatisticamente significativa com 0 abandono do hábito de fumar, durante a gestação, foram a renda familiar, o hábito de fumar da mãe e do companheiro, a idade com que a mulher iniciou a fumar e a duração e intensidade do hábito. A renda familiar apresentou uma importante associação direta com a interrupção do hábito de fumar durante a gravidez. Outros estudos confirmam esta associáção. Uma reanálise dos dados de Victora e col. ${ }^{15}$ detectou probabilidade $60 \%$ maior de abandono do hábito de fumar quando parturientes com renda familiar mensal de 6,1-10 salários mínimos eram comparadas com as de renda menor ou igual a um salário $(R P=1,61$; IC 95\% 1,12-2,31). Da mesma forma, Kuzma e Kissinger ${ }^{9}$ encontraram uma associação negativa entre o nivel socioeconômico (educação e renda) e a continuação do hábito de fumar durante a gravidez.

Outras variáveis sociodemográficas como idade, escolaridade, ausência do companheiro e aglomeração doméstica não apresentaram associação com a modificação do hábito durante a gravidez. A reanálise dos dados de Victora e col. ${ }^{15}$, no entanto, detectou associação linear inversa $(p=0,0001)$ entre abandono do hábito na gravidez e escolaridade, sendo a taxa de abandono de $30 \%$ entre mulheres com 9 anos ou mais de escola contra $17 \%$ entre aquelas sem escolaridade formal. A probabilidade de abandono foi $77 \%$ maior entre mulheres com 9 anos ou mais de escolaridade comparadas com as analfabetas ( $R P=1,77$; IC 95\% 1,17-2,69). Da mesma forma, naquele estudo, a presença do companheiro esteve significativamente associada com o abandono ( $p<0,001$ ): $23 \%$ das mulheres que viviam com companheiro pararam de fumar contra $18 \%$ nas demais.

O hábito de fumar do companheiro, por outro lado, apresentou associação estatisticamente significativa com a evolução do hábito, no presente estudo. As mulheres cujo marido ou companheiro não fumavam tiveram $43 \%$ mais chance de abandonar o hábito do que as demais.

O hábito de fumar da mãe da gestante também representou um importante fator prognóstico: mulheres cujas mães não tinham história de tabagismo (atual ou no passado) apresentaram probabilidade $60 \%$ maior de abandonar quando comparadas com as filhas de mães fumantes.

Assim, o hábito de fumar da mãe e do companheiro representaram ambos, importantes fatores de risco para o tabagismo no início e fatores prognósticos para o abandono do mesmo durante a gestação. Não foi possível localizar na literatura dados comparativos provenientes de outras fontes.
Três variáveis relativas ao hábito de fumar (idade de início, duração e intensidade) estiveram significativamente associadas com a taxa de abandono. As mulheres que iniciaram o hábito antes dos 14 anos de idade tiveram 57\% menos chance de parar durante a gravidez. Da mesma forma, a taxa de abandono apresentou associação linear inversa com a duração do hábito: quanto menor a duração, maior a probabilidade de interrupção. Quanto à intensidade do hábito, 53\% das mulheres que fumavam de um a 5 cigarros por dia no início da gravidez pararam de fumar, contra $33 \%$ das que fumavam mais de 10 . Os achados referentes ao número de cigarros são consistentes com os relatados por Sexton e Hebel ${ }^{13}$ em que $71 \%$ das mulheres que fumavam menos de 10 cigarros por dia no início da gravidez interromperam o hábito espontaneamente durante a gravidez, contra $17 \%$ das que fumavam mais de 10 .

A redução do número de cigarros consumidos por dia entre as gestantes que continuaram fumando é consistente com os resultados de Rubin e col. ${ }^{12}$ que detectaram uma média de 9,0 cigarros por dia durante a gravidez $(s=5,8)$ e os de Sexton e Hebel ${ }^{13}$ que verificou uma redução significativa na quantidade de cigarros fumados até a $18^{2}$ semana gestacional.

\section{Conclusóes}

A presente investigação revelou que a baixa escolaridade e o hábito de fumar da mãe e do companheiro da gestante são importantes fatores de risco para o tabagismo no início da gravidez. Por outro lado, a baixa renda familiar, o hábito de fumar da mãe e do companheiro e as características do hábito da mulher, particularmente a idade mais precoce de início e a maior duração e intensidade, representam fatores prognósticos importantes para a interrupção do tabagismo durante a gravidez.

Com base nos presentes achados, sugere-se que os programas de intervenção que visem à interrupção do hábito de fumar durante a gestação devem concentrar esforços em gestantes mais pobres, companheiras e filhas de fumantes, que fumem mais de cinco cigarros por dia, cujo hábito tenha iniciado antes dos 14 anos de idade e que dure mais de cinco anos.

HALAL, I. S. et al. [Determining factors related to smoking and its abandonment during pregnancy in an urban locality in Southern Brazil]. Rev. Saúde Pública, 27: 105-12, 1993. A cross-sectional study of 873 pregnant women attending a prenatal care clinic was undertaken in Pelotas (Southem Brazil), over a one-year pe- 
riod (1989-90) for the purpose of identifying possible risk and prognostic factors for smoking during pregnancy. The prevalence of smoking at the beginning of pregnancy was $40.8 \%$. The smoking habits of the woman's mother and partner, as well as her educational level, were associated with smoking at the beginning of pregnancy. Smoking in the partner was associated with an approximately two-fold increase in the risk of smoking. The smoking interruption rate, until the 15 th-22nd gestational week, was of $35.6 \%$. Family income, smoking habits of woman's mother and partner, age at starting, duration and intensity of the woman's habit were all associated with the interruption during pregnancy. The above results were not affected by adjustment for confounding variables made through stratified analysis.

Keywords: Smoking, epidemiology. Pregnancy. Socioeconomic factors.

\section{Referências Bibliográficas}

1. BARIC, L. \& MaCARTHUR, C. Health norms in pregnancy. Br. J. Prev. Soc. Med., 31: 30-8, 1977.

2. BARROS, F. C.; VICTORA, C. G.; HUTTLY, S. R. A.; KIRKWOOD, B. R.; VAUGHAN, J. P. Comparison of the causes and consequences of prematurity and intrauterine growth retardation: a longitudinal study in southem Brazil. Pedialrics, 90(2): 1-7, 1992

3. BERKOWTTZ, G. S.; HOLFORD, T. R.; BERKOWITZ, R. L. Effects of cigarette smoking, alcohol, coffee and tea consumption on preterm delivery. Early Hum. Dev., 7: 239-50, 1982.

4. BORLAND, B. \& RUDOLPH, J. P. Relative effects of low socio economic status, parental smoking and poor scholastic performance on smoking among high school students. Soc. Sci. Med., 9: 27-30, 1975.
5. FERRAZ, E. M.; GRAY, R. H.; CUNHA, T. M. Determinants of preterm delivery and intrauterine growth retardation in North-East Brazil. Int. J. Epidem., 19: 101-8, 1990.

6. HORN, D; COURTS, F. A.; TAYLOR, R. M.; SOLOMON, E. S. Cigarette smoking among high school students. Am. J. Public Health, 49: 1497-511, 1959.

7. KIRKWOOD, B. R. Essencials of medical statistics. Oxford, Blackwell Scientific Publication, 1988.

8. KRAMER, M. S. Determinants of low birth weight: methodological assessement and meta-analysis. Bull. World Health Organ., 65: 663-737, 1987.

9. KUZMA, J. W. \& KISSINGER, D. G. Patterns of alcohol and cigarette use in pregnancy. Neurobehav. Toxicol. Teratol, 3: 211-21, 1981.

10. LILLEY, J. \& FORSTER, D. P. A randomized controlled trial of individual counselling of smokers in pregnancy. Public Health, 100: 309-15, 1986.

11. MARTLN, J. C. An overview: matemal nicotine and caffeine consumption and offspring outcome. Neurobehav. Toxicol. Teralol., 4: 421-7, 1982.

12. RUBIN, D. H.; KRASILNIKOFF, P. A.; LEVENTHAL, J. M.; WERLE, B.; BERGET, A. Effect of passive smoking on birth weight. Lancet, $2: 415-7,1986$.

13. SEXTON, M. \& HEBEL, J. R. A clinical trial of change in matemal smoking and its effect on birth weight. JAMA, 251: $911-5,1984$.

14. TERRIN, M \& MEYER, M. B. Birth weight-specific rates as a bias in the effects of smoking and other perinatal hazards. Obstet. Gynecol., 5: 636-8, 1981.

15. VICTORA, C. G.; BARROS, F. C.; VAUGHAN, J. P. Epidemiologia da desigualdade. São Paulo, Hucitec, 1988.

16. WINDSOR, R. A.; CUTTER, G.; MORRIS, J.; REESE, Y; MANZELLA, B.; BARTLETT, E. E.; SAMUELSON, C.; SPANOS, D. The effectiveness of smoking cessation methods for smokers in public health matemity clinic: a randomized trial. Am.J. Public Health, 75: $1389-92,1985$.

Recebido para publicação em 9.11.1992 Aprovado para publicaçāo em 92.1993 